Ebisu Ebisu

Études japonaises Études japonaises

57 | 2020

Les architectes de l'ère Heisei (1989-2019). Rôles, statuts, pratiques et productions

\title{
Disparitions et émergences
}

Sylvie Brosseau

(2) OpenEdition

Journals

Édition électronique

URL : http://journals.openedition.org/ebisu/5421

DOI : 10.4000/ebisu.5421

ISSN : 2189-1893

Éditeur

Institut français de recherche sur le Japon à la Maison franco-japonaise (UMIFRE 19 MEAE-CNRS)

Édition imprimée

Date de publication : 15 décembre 2020

Pagination : 395-418

ISSN : $1340-3656$

Référence électronique

Sylvie Brosseau, «Disparitions et émergences », Ebisu [En ligne], 57 | 2020, mis en ligne le 20 décembre 2020, consulté le 29 mars 2021. URL : http://journals.openedition.org/ebisu/5421 ; DOI : https://doi.org/10.4000/ebisu.5421 


\section{Conclusion : disparitions et émergences}

En trente ans, le cours normal des choses entraîne des pertes, des remplacements, des émergences. Exister puis disparaître, ainsi va la loi du devenir. Cependant, à la lecture de ce dossier sur l'architecture de l'ère Heisei, la disparition, de diverses manières, devient un phénomène récurrent : destruction de bâtiments, de quartiers, de villes; extinction de leurs habitants; abandon, rétraction ou effacement, sous des formes et dans des espaces variés. Quelque chose émerge-t-il malgré tout? Est-il possible d'en saisir les traces à travers les différents textes?

\section{Cataclysmes}

D'abord, bien sûr, ressortent au premier plan toutes les destructions causées par les puissants séismes qui ont frappé le Japon - Kobe en 1995, puis le littoral du Tōhoku en 2011 - celui-ci complexifié à l'infini par la catastrophe nucléaire de Fukushima. De multiples autres secousses, oubliées depuis longtemps en dehors de leur échelle locale, continuent de peser sur le quotidien de beaucoup de gens. Pendant les trente années de l'ère Heisei 平成 (1989-2019), les désastres sismiques ont anéanti plusieurs centaines de milliers de constructions de toutes sortes, et fait 30000 victimes au total. En outre, la violence des cieux - typhons, tornades, pluies torrentielles - a provoqué la destruction de dizaines de milliers de bâtiments, 
principalement des maisons, et la mort ou disparition d'un millier de personnes ${ }^{1}$. Le but ici n'est pas de faire une exhaustive liste macabre, mais si l'on compare avec les époques précédentes, Heisei ne fut vraiment pas une ère ménagée par les forces de la nature : vingt-quatre séismes destructeurs et dix-neuf épisodes météorologiques extrêmes se sont abattus sur le Japon pendant cette période 2 . On peut encore ajouter deux éruptions volcaniques meurtrières ${ }^{3}$ : en 1991, durant plusieurs mois, les nuées ardentes dévalant le mont Unzen dans le département de Nagasaki ont enseveli quarante-trois personnes et une vallée; en octobre 2014, une explosion soudaine sur le mont Ontake dans le département de Nagano a fait soixante-trois victimes parmi les nombreux randonneurs surpris sur les pentes. Pour mémoire, la précédente éruption mortelle au Japon datait de 1958, et avait fait douze victimes lors d'une explosion du mont Aso (département de Kumamoto).

Ce que l'on constate, c'est que les conséquences de ces phénomènes, de moins en moins naturels, sont amplifiées ou provoquées par les actions humaines : urbanisation dans des zones submersibles; abandon ou exploitation inadéquate du couvert forestier, causes de glissements de terrain ou d'effets de barrage qui se rompent brutalement; entretien insuffisant des digues, des tunnels, des ouvrages d'art, toujours plus nombreux et complexes; construction sur les sols non stabilisés des comblements artificiels du littoral qui se liquéfient en cas de secousse sismique; développement d'activités touristiques dans des zones sensibles telles que les pentes des volcans, entre autres. Au-delà des données chiffrées, ces événements meurtrissent les territoires, ébranlent les collectivités locales, et touchent la société dans

1. Exemple le plus dévastateur de la période : du 28 juin au 8 juillet 2018 , des pluies torrentielles se sont abattues sans interruption sur tout le Japon. Particulièrement violentes dans l'Ouest du pays, elles y ont provoqué la mort de 232 personnes, la destruction totale de près de 7000 maisons, et plus de 21000 bâtiments ont subi des dégâts.

2. “Saigai o motarashita kishō jirei » 災害をもたらした気象事例 (Événements météorologiques causes de désastres), Kishōchō 気象庁 (Agence de la météorologie nationale) : https://www.data.jma.go.jp/obd/stats/data/bosai/report/index.html et Naitō Hiroshi + Nikkei Ākitekuchua 内藤廣十日経アーキテクチュア, Kenshō. Heisei kenchikushi 検証-平成建築士 (Examen-Histoire de l'architecture de Heisei), Tokyo, Nikkei Business Publications, 2019, p. 110-111.

3. “Kako ni hassei shita kazan saigai » 過去に発生した火山災害 (Historique des catastrophes d'origine volcanique), Kishōchō: https://www.data.jma.go.jp/svd/vois/data/ tokyo/STOCK/kaisetsu/volcano_disaster.htm. 
son ensemble, ce qui motive sans doute le recours croissant au concept de résilience (rejiriensu レジリエンス) appliqué de plus en plus largement ${ }^{4}$, une façon de nommer un état d'exception qui devient le normal ${ }^{5}$.

$\mathrm{Au}$ Japon, les séismes importants entraînent la révision des normes, des lois, des pratiques, et ces évolutions concernent en première ligne la construction, donc les architectes. Itō Toyō* 伊東豊雄 (né en 1941) est un de ceux qui a exprimé clairement ses propres remises en cause après mars $2011^{6}$. D'autres intègrent à leurs projets la possibilité du cataclysme, audelà de la sécurité des calculs de structure, en prenant en compte la fonction de refuge par défaut qu'ont au Japon les grands équipements couverts. Par exemple, Naitō Hiroshi* 内藤廣 (né en 1950), à l'occasion d'un projet pour un vaste gymnase à Kusanagi, dans le département de Shizuoka (achevé en 2015), a imaginé les habitants, réfugiés potentiels, voire sinistrés, qui passeront peut-être des jours, des semaines, à regarder le plafond; il a dessiné une toiture dont la structure laisse passer la lumière, tamisée par un plafond de lames en bois qui réunit protection et contemplation ${ }^{7}$.

\section{Démolitions}

À ces destructions majeures, non voulues mais dans lesquelles les choix humains pèsent d'un poids toujours plus grand, s'ajoutent les démolitions décidées, précédant une reconstruction. C’est le mécanisme du scrap and build évoqué ou décrit dans plusieurs textes, la logique du capitalisme à l'œuvre dans les villes, particulièrement visible au Japon où le frein du patrimoine historique ou des pesanteurs culturelles ne ralentit pas le cycle des démolitions/

4. Scoccimarro Rémi, " 11 mars 2011 : de la vie en préfabriqués à l’assignation à résilience ", in Doumet Christian \& Ferrier Michaël (dir.), Penser avec Fukushima, Nantes, Cécile Defaut, 2016, p. 133-159.

5. Bizet François, "L'inhabitat ", in Doumet \& Ferrier 2016, op. cit., p. 161-190.

6. Itō Toyō, L'Architecture du jour d'après, Bruxelles, Les Impressions Nouvelles, 2014.

7. Grout Catherine, "L'architecture comme expérience sensorielle, culturelle et sociale. Au sujet de quelques projets de Naito Hiroshi ", Journal des anthropologues, $\mathrm{n}^{\circ} 134-135,2013$, p. 120.

* Les mots suivis d'un astérisque renvoient au lexique situé à la fin du dossier. 
reconstructions. Aujourd'hui, certains des bâtiments les plus représentatifs de l'époque de la bulle financière, qualifiés d'iconiques, expressions d'une créativité sans entrave (ni financière, ni esthétique, ni culturelle), ont souvent déjà été remplacés. Cette " architecture comme une image ${ }^{8}$ " telle que la nomme Itō, achevée au début de l'époque Heisei, qui a fait l'objet de publications dans des revues internationales, a été exposée dans de grands musées, a en partie disparu. Certains de ces bâtiments-objets, dessinés par des architectes de renom ou en vogue, s'érigeaient au pic de la bulle sur des terrains si étroits qu'ils étaient appelés empitsu biru 鉛筆ビル (immeubles crayons) à cause de leur forme. Ils agglomèrent boutiques, restaurants, salles de sport, show-rooms, et sont des lieux clos de déambulation commerciale. Connus par le nom qui leur est attribué, qui permet d'être repérés et cités dans les magazines, ils se sont multipliés, transformant certains quartiers de Tokyo, tel Aoyama, en exposition de pavillons. L'architecture est considérée comme un objet de consommation, elle se consomme, se consume et Itō constatait en 1991 qu' elle « s'assimile à la mode, et la figure de l'architecte ne se distingue guère plus de celle du graphiste ou du publicitaire ${ }^{9}$ ». À l'apogée de la bulle, au début de l'ère Heisei, le jugement sur la ville japonaise, particulièrement Tokyo devenue puissante, s'inverse. Ce qui était considéré comme laideur, désordre, devient " anarchie progressive ", chaos adaptatif d'une "ville amibe " et fascinant ${ }^{10}$. L'éclatement de la bulle a effectué une sélection, in fine une montée en gamme, et l'avenue Omotesandō est devenue elle-même un immense show-room des grandes marques du luxe en parade derrière les façades de somptueux bâtiments dessinés par les stars de l'architecture mondiale des années 2000. Ce n'est plus seulement le jugement mais le modèle qui s'est inversé : les Champs-Élysées se réferent aujourd'hui à Omotesandō et non plus l'inverse, la ville japonaise a exalté un nouveau paradigme, celui du triomphe du " néolibéralisme sur le sol ${ }^{11}$ ", de la globalisation.

8. Itō, op. cit., p. 118.

9. Brosseau Sylvie, "L'architecture moderne au Japon ", in Ouvrage collectif, Le Japon 1994, Tokyo, Maison franco-japonaise, 1994, p. 335.

10. Berque Augustin, "La ville japonaise en quête d'un paradigme ", Lumières de la ville, $\mathrm{n}^{\circ} 1,1990$, p. $40-47$.

11. Voir actuellement les différents travaux sur ce thème : Biau Véronique, Les Architectes au défi de la ville néolibérale, Marseille, Parenthèses, 2020; Cupers Kenny, Mattsson Helena \& Gabrielsson Catharina (dir.), Neoliberalism on the Ground. Architecture and Transformation from the 1960s to the Present, Pittsburgh, University of Pittsburgh Press, 
À Tokyo, quelques-uns des immeubles phares de cette époque de la bulle subsistent, par exemple Spiral conçu par Maki Fumihiko* 槇文彦 (né en 1928), M2 par Kuma Kengo* 隈研吾 (né en 1954) (fig. 01), Collezione par Andō Tadao* 安藤忠雄 (né en 1941), mais à Osaka, les plus fameux de ceux conçus par Takamatsu Shin* 高松伸 (né en 1948), minutieusement dessinés par l'architecte, qui avaient particulièrement retenu l'attention à l'époque (voir les monographies, les expositions, les commentaires évoqués par Benoît Jacquet et Yann Nussaume), ont été démolis à partir des années 2000. Ces réalisations emboîtaient, selon des symétries appuyées, des formes opaques d'une puissante matérialité assumée, en quête d'effets visuels forts, encore plus marqués la nuit grâce à la lumière. Se superposaient des images de machines ou de paysages urbains comme issues des mondes cyberpunks que l'on trouve dans des mangas ou des dessins animés ${ }^{12}$.

Une remarque : l'esthétique architecturale est souvent discutée de façon autoréférentielle, sans sortir du champ de l'architecture, éventuellement par rapport à la nature, rarement dans les cadres de références plus larges des cultures visuelles. Depuis cette période de gloire, Takamatsu s'est lui aussi rallié au blanc, au verre, derrière lequel transparaît parfois le bois, soit le vocabulaire consensuel de l'architecture actuelle. Kuma, quant à lui, a fait un saut périlleux, remarque ironiquement Naitō (fig. 01 et 02 ).

2020 ; Ernwein Marion, Les Natures de la ville néolibérale. Une écologie politique du végétal urbain, Grenoble, UGA, 2019.

12. Je pense particulièrement à Akira アキラ, manga écrit et dessiné par Ōtomo Katsuhiro 大友克洋 de 1982 à 1990, adapté en dessin animé par l'auteur en 1988, sans grand succès avant sa sortie en VHS. La première image du film montre un plan du Néo-Tokyo post-apocalyptique, cadre des événements décrits, où la baie est en grande partie comblée et construite. L'action est située en 2019, et parfois apparaît un panneau avec le décompte des jours avant les JO qui doivent se dérouler en 2020, mais la catastrophe finale laisse penser que ces JO n'auront jamais lieu. La ville est le lieu dystopique de confrontations entre divers groupes de protagonistes dont une secte religieuse qui évoque de façon étonnante la secte Aum (Oumu Shinrikyō オウム真理教). Les hautes tours rutilantes du Néo-Tokyo érigé dans la baie, contrastent avec la vieille ville, en partie abandonnée, où les laissés-pour-compte survivent. Le paysage urbain reflète les écarts sociaux creusés. Ce film est sorti en 1989 aux États-Unis puis en Europe, où il devient culte. Il exerce toujours de très fortes influences revendiquées par les artistes de divers genres des cultures populaires. Akira est ressorti en France en 2020 et ce film est en train d'être découvert par la génération actuelle des jeunes de 20 ans, aussi bien au Japon que dans le monde. 


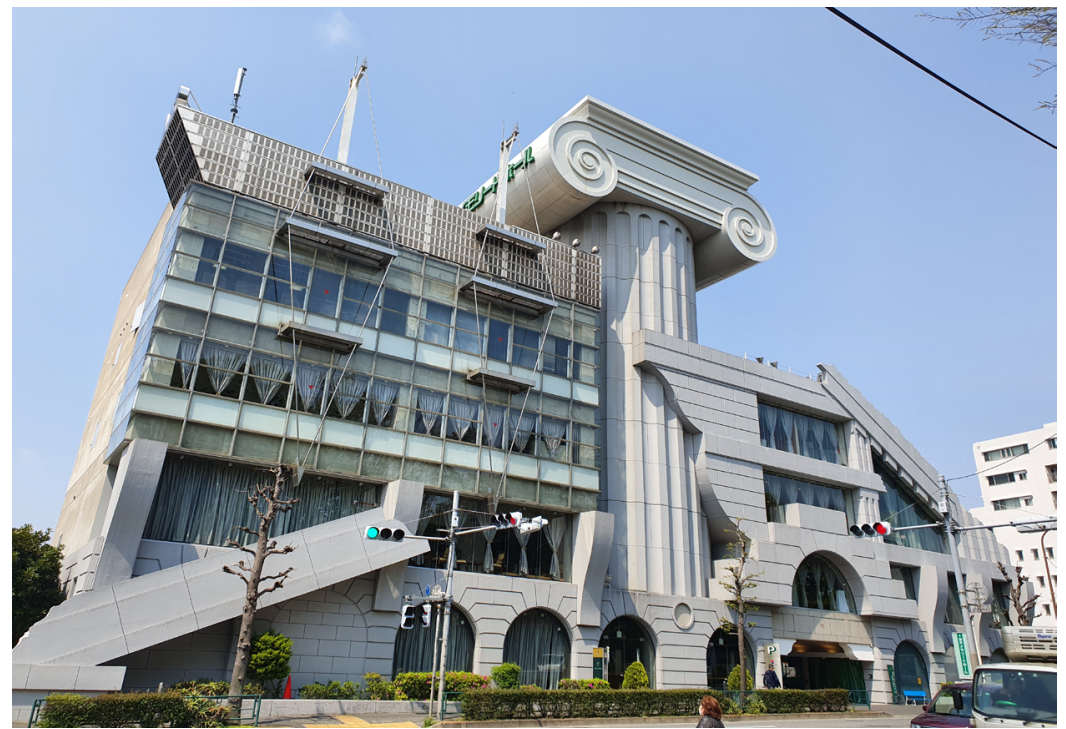

Fig. 01

M2, arrondissement de Setagaya, Tokyo, 1991, par Kuma Kengo.

Photo : domaine public.

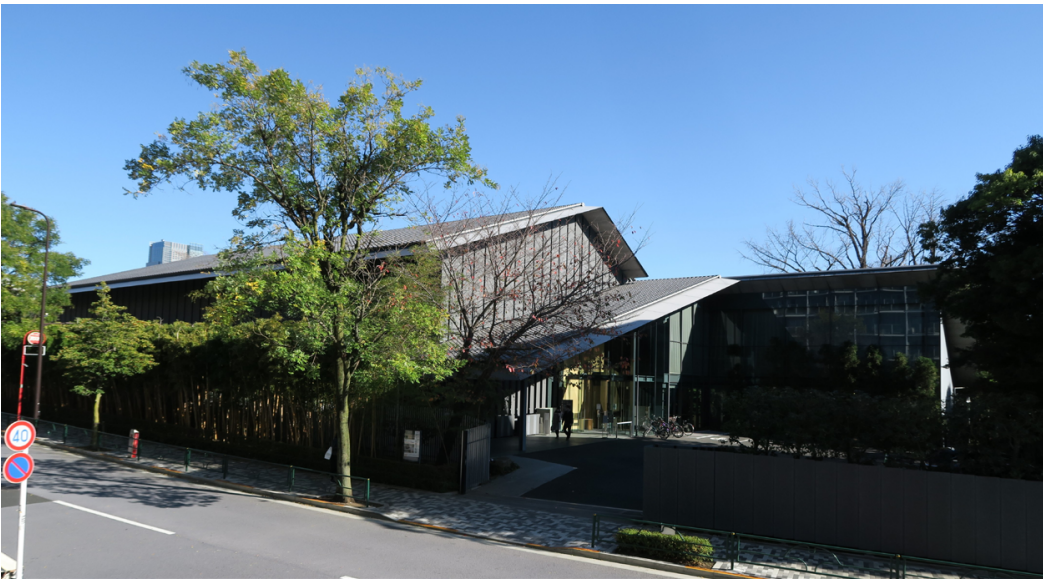

Fig. 02

Nezu bijutsukan 根津美術館 (musée Nezu), Tokyo, 2009, par Kuma Kengo.

Photo: Sylvie Brosseau 2020. 


\section{Vacance}

Les cataclysmes ne sont pas les seules causes de destructions. Le temps qui passe et les météores disloquent assez vite les maisons en bois si elles restent vacantes trop longtemps. De plus en plus nombreuses, elles sont les témoignages matériels et visibles des rétractions régionales et démographiques qui se cumulent. Lorsqu'on se promène, à la campagne, dans les petites villes provinciales, et maintenant aussi dans certains quartiers périphériques de Tokyo, on rencontre ces maisons abandonnées, envahies de végétation, à la toiture en train de percer, puis aux murs s'écroulant. Les akiya 空き家, maisons vacantes, font maintenant partie du paysage, et laissent un sentiment de désolation et d'interrogation sur le sens du développement qui continue à prôner la croissance. Tout un vocabulaire se développe durant l'ère Heisei pour nommer ces phénomènes de dépérissement ${ }^{13}:$ shurinkingu shiti シュリンキング・シティ (shrinking city), toshi no shukushō 都市の 縮小 (déclin urbain), suponjika スポンジ化 (évidement en éponge), akiya mondai 空き家問題 (question de la vacance). Les logements vacants sont devenus un problème car ils finissent par représenter un danger pour le voisinage ou le passage, et sont un souci des collectivités locales qui cherchent des moyens et des solutions pour pouvoir intervenir. De 9,4\% en 1989, le taux de vacance est passé à 13,6\% en 2018, avec de fortes disparités régionales : les départements les plus concernés sont Yamanashi $(21,3 \%)$ qui jouxte Tokyo, Wakayama (20,3\%) et Nagano (19,5\%), où un logement sur cinq est vacant. Les moins touchés sont Saitama (10,2\%) qui jouxte Tokyo aussi, Okinawa $(10,2 \%)$ et Tokyo $(10,6 \%)^{14}$. Ce ne sont donc pas les départements les plus pauvres ou les plus éloignés de l'aire de la capitale qui ont les taux de vacance les plus élevés. Au Japon, les habitants s'éteignent et les maisons se vident.

13. Buhnik Sophie, "Shukushō 縮小 la rétraction urbaine", in Bonnin Philippe, Nishida Masatsugu \& Inaga Shigemi (dir.), Vocabulaire de la spatialité japonaise, Paris, CNRS Éditions, 2014, p. 463-466.

14. “Akiya-ritsu, kako saikō no 13,6 \% toshi-bu de takai chiiki mo " 空き家率、過去最高 の $13.6 \%$ 都市部で高い地域も (Taux de vacance à un niveau historique de 13,6 \%, même dans les quartiers chers des villes), Nihon keizai shinbun 日本経済新聞, 26 avril 2019. 


\section{Disparition d'un maître}

La revue Nikkei Architecture ${ }^{15}$ a publié en avril 2019 un numéro spécial sur l'architecture de l'ère Heisei ${ }^{16}$, dans lequel une longue conversation avec l'architecte Naitō sert de fil conducteur entre différentes thématiques examinées. La revue a également demandé à vingt personnalités d'horizons divers dans le monde de la construction (chercheurs et/ou praticiens, nés de 1937 à 1979, dont une seule femme), de choisir chacun les dix projets qui leur semblent emblématiques de la période Heisei. Plus que le palmarès, ce qui étonne dans la liste ainsi établie, c'est la disparition d'une figure, celle d'Andō Tadao, au regard des importants projets qu'il a réalisés et de son rôle éminent sur la scène architecturale, au Japon et dans le monde. Andō est sans doute l'architecte japonais le plus connu à l'étranger actuellement, en tout cas en France : il a bénéficié, entre autres, de deux expositions au Centre Pompidou (1993 et 2018), et il est l'architecte désigné par la Fondation Pinault pour la réhabilitation des prestigieux bâtiments qui exposent ses collections : le Palazzo Grassi (2006) et la Punta della Dogana (2009) à Venise, puis la Bourse de Commerce (2021) à Paris. Au Japon, il est l'architecte choisi en 1989 par la fondation Benesse pour établir sur l'île de Naoshima située dans la mer Intérieure, divers musées, lieux d'accueil et hôtels, soit un ensemble de projets qui a transformé l'île en un centre d'art d'influence et de renommée internationales. L'architecture et le paysage scénographient un processus de sacralisation de la culture et l'île est devenue un modèle idéalisé de revitalisation locale.

Andō est apparu au cours de l'ère Heisei tel l'architecte officiel du Japon, notamment après la disparition de Tange Kenzō* 丹下健三 (1913-2005)

15. La revue Nikkei Architecture (Nikkei Äkitekuchua 日経アーキテクチュア), créée en 1976, traite du monde de la construction et de tous ses acteurs en mettant en avant l'innovation. Cette revue en ligne est éditée par Nikkei Business Publications qui fait paraître le Nihon keizai shinbun, abrégé en Nikkei, journal économique diffusant le Nikkei 125, principal indice boursier du Japon. NKS a également développé un réseau de chaînes de télévision dont la plus importante est TV Tokyo, et est également propriétaire du Financial Times depuis 2015.

16. Le titre est Kenshō-Heisei kenchikushi. Naitō Hiroshi + Nikkei Ākitekuchua 検証平成建築士 内藤廣十日経アーキテクチュア (Examen-Histoire de l'architecture de Heisei. Naitō Hiroshi + Nikkei Architecture). 
en 2005 dont il prend la place symbolique. Maître d'œuvre du pavillon du Japon à l'Exposition universelle de Séville en 1992, il a reçu de multiples récompenses et décorations, dont le prix Pritzker* en 1995, et il est nommé président du jury pour le concours du stade olympique en 2016. Son travail a fait l'objet d'une grande rétrospective en 2017 au Nouveau musée national de Tokyo (Kokuritsu shinbijutsukan 国立新美術館).

Dans le classement évoqué plus haut, parmi les vingt experts disposant chacun de dix choix, seules sept personnes ont désigné une réalisation d'Andō ${ }^{17}$. C'est presque autant que le projet du stade olympique conçu par Zaha Hadid* (1950-2016), choisi en hommage et aussi parce que le fiasco de ce concours dont le résultat a été annulé au bout de deux ans, semble représentatif des dysfonctionnements de l'époque. Une gêne vis-à-vis de l'architecte décédée brutalement peu après cette éviction grossière, quoi qu'on pense du projet, est perceptible à travers plusieurs textes. Les choses se seraient-elles déroulées ainsi s'il avait été signé par un homme?

Toujours dans ce classement, à l'opposé, Itō est retenu par dix-huit personnes sur vingt, dont une qui a même cité deux de ses réalisations. Itō et des architectes très proches de lui, appartenant à sa lignée telle que la décrit Olivier Meystre, exercent une force d'attraction claire et évidente, ce sont eux qui captent toute l'attention. Parmi les dix projets les plus cités ${ }^{18}$, en première place, élue presque unanimement, se hisse la médiathèque de Sendai* conçu par Itō; en deuxième vient le musée du Xxi ${ }^{\mathrm{e}}$ siècle* à Kanazawa, par SANAA*; puis en $4^{\mathrm{e}}$ place à égalité, le bâtiment universitaire dessiné par Ishigami Jun.ya* 石上純也 (né en 1974), et le musée de Teshima* par Nishizawa Ryūe* 西沢立衛 (né en 1966), exploré et restitué par François Bizet dans ce numéro. Ces quatre bâtiments, par leur luminosité, leur parti minimal, tendent vers des formes d'effacement (offrent des pauses dans notre monde désaccordé?).

Après la bulle, toute une génération a aspiré à une architecture dotée d'une apparence de simplicité, voire de platitude, de banalité. Ce

17. Trois fois Hikari no kyōkai 光の教会 (L'Église de lumière, 1989), à Osaka où le mur pignon en béton derrière l'autel est percé dans sa hauteur et sa largeur entières d'étroites ouvertures formant une croix; et quatre fois un projet à Naoshima.

18. Voir l'article de Funo Shūji dans ce numéro, p. 76. 
"dépouillement maniériste de l'enveloppe " qualifié de flat, voire de super$f_{a t}{ }^{19}$, qu'enveloppe-t-il? Les parois ultraminces enclosent des espaces aux affectations floues ou flottantes ${ }^{20}$. À propos du musée de Kanazawa, de l'architecture publique dessinée par SANAA, voire même de la médiathèque de Sendai, Naitō parle de culture du konbini コンビニ, de konbinika コンビニ化 (konbinisation) : tel un convenience store, ces bâtiments n'intimident pas, n'imposent rien, on peut entrer et ressortir sans rien faire ou pas grandchose, ils sont sans spécificités fortes mais on peut y trouver une satisfaction. À «l'ère des boîtes sans qualitée ${ }^{1}$ ", l'architecture ne nous déconcerte pas trop, elle ne demande pas trop d'effort, elle est là pour nous laisser venir. Rien de massif ou d'imposant, le contraire d'Andō, de son architecture de murs pleins et de béton brut, qui devient coercitive à force de dicter des sensations, d'orienter notre regard vers une unique ouverture dans une paroi pleine, de diriger notre corps par un escalier monumental ou un couloir étroit. À l'inverse, l'architecture de l'effacement repose notre attention et nos sens fatigués d'images, éveille peut-être des sensations autres que visuelles, plus primitives ou originelles? Après la médiathèque de Sendai, les détails disparaissent toujours plus.

\section{Disparition des revues}

L'architecture est pratique, et aussi discours, réflexion théorique, mais durant l'époque Heisei, ont aussi disparu au Japon plusieurs grandes revues d'architecture dont des publications historiques, un fait remarqué par Olivier Meystre et Naitō Hiroshi.

Notamment, Kenchiku bunka 建築文化 (Culture de l'architecture), revue créée en 1946 dont le dernier numéro a été publié en décembre 2004. Cette revue présentait l'architecture non pas comme objet mais comme culture, avec le dessein d'approfondir la réflexion sur les liens entre la société et l'architecture, et de contrebalancer Shinkenchiku 新建築 (Nouvelle

19. Tardits Manuel, Tôkyô. Portraits \& Fictions, Blou, Le Gac Press, 2011, p. 295-296; Naitō + Nikkei, op. cit., p. 210.

20. Tardits, op. cit., p. 299.

21. Ibid., p. 300. 
architecture), qui tous les mois publie de façon très factuelle, des photos et des données sur les constructions achevées.

SD (Space Design), revue mensuelle, a été créée en 1965 par les éditions Kajima shuppankai 鹿島出版会, fondées en 1963 par le PDG de l'entreprise de construction Kajima, un des cinq super zenekon* ゼネコン, et était consacrée à l'histoire et aux recherches architecturales. Le dernier numéro est celui de décembre 2000, même si les éditions SD continuent de publier des ouvrages sur l'architecture.

Un seul groupe, Shinkenchiku-sha 新建築社, publie aujourd'hui les principales revues d'architecture, accessibles en ligne depuis 2017, dont : Shinkenchiku, créée en 1925, revue mensuelle qui présente les projets récents au Japon, ou à l'étranger si l'architecte est japonais; JA (The Japan Architect), mensuel entièrement bilingue japonais/anglais créé en 1956, devenu trimestriel en 1991, avec l'objectif de faire connaître l'architecture japonaise à l'étranger; $a+u$ (architecture + urbanism), revue bilingue japonais/anglais créée en 1971 dont le but est également d'insérer le Japon dans le réseau international de l'architecture. Ce groupe publie aussi depuis 1985 Jūtaku tokushū 住宅特集, qui présente des maisons dessinées par des architectes avec de très belles photos et des plans. Pour Naitō, cette source éditoriale quasi unique (avec Nikkei Architecture) produit une situation qu'il compare au Jimintō 自民党 (Parti libéral démocrate) qui n’a plus actuellement d'opposition, ce qui lui semble être un appauvrissement de la culture architecturale $^{22}$. Alors que des lieux d'exercice de la pensée ont disparu, il remarque le contraste avec la brillante réussite de Casa BRUTUS, magazine facile, séduisant, sexy, mais qui n'a rien à voir avec une revue d'architecture. C'est la victoire de l'image sur les mots, de l'entertainment sur la réflexion. Avec Casa BRUTUS, l'architecture est considérée comme une ressource profitable, support chic à la consommation de tout un ensemble de choses à la mode.

Subsiste un autre éditeur spécialisé dans la présentation de l'architecture sous l'angle photographique et esthétique, et qui a joué un rôle essentiel dans la diffusion de l'architecture japonaise à l'étranger, comme dans la redécouverte visuelle de l'œuvre de certains architectes occidentaux, tels Antonio Gaudi (1852-1926) ou Franck Lloyd Wright (1857-1959). ADA

22. Naitō + Nikkei, op.cit., p. 67-69. 
Edita Tokyo, fondé en 1970 par le photographe et critique d'architecture Futagawa Yukio 二川幸夫 (1932-2013), publie diverses séries sous le label GA (Global Architecture) et gère à Tokyo, dans l'arrondissement de Shibuya, une galerie avec une librairie consacrée à l'architecture. En 1992 est lancé le mensuel GA Japan, consacré uniquement à l'architecture japonaise, thématique sur laquelle s'est recentré GA au cours de l'ère Heisei.

\section{Émergence d'une génération qui fabrique}

Funo Shūji semble penser que le moteur à deux temps du renouvellement urbain au Japon, le scrap and build, est en train de s'enrayer dans son mouvement. Naitō ne le pense pas car d'après lui, le monde de la construction n'a guère changé, si ce n'est une concentration toujours plus importante de la puissance. Cependant, même si la durée de vie d'une maison semble toujours brève à Tokyo, en moyenne environ 25 ans, et que la médiocre qualité générale de la construction ne permet guère plus, les architectes, les habitants, les villes semblent découvrir les vertus et richesses de la réhabilitation des bâtiments existants.

La gare de Tokyo constitue un exemple emblématique et exceptionnel (fig. 03). Celle-ci a, pendant longtemps, été considérée surtout comme une opportunité foncière exceptionnelle qui a suscité de nombreux projets successifs de tours. Cependant, elle est finalement restée dans sa volumétrie d'origine. En partie reconstruite à l'identique plutôt que restaurée, elle a conservé son profil peu élevé et sa façade familière du côté de Marunouchi, et les deux rotondes à verrières qui articulent les multiples entrées et fonctions de la gare, ont gardé leur forme et leur dessin d'origine ainsi que leur rôle. Cette réhabilitation, une opération d'un type courant en Europe, au cour de Tokyo a acquis une signification très importante pour le monde de l'architecture japonais. Elle a été élue dans le classement susmentionné des dix projets représentatifs de l'époque Heisei. C'est autour de la gare que les tours se concentrent, toujours plus hautes, avec un nouveau projet en cours, la Torch Tower, construite par Mitsubishi, qui sera le plus haut bâtiment du Japon (390 mètres avec 63 étages). 


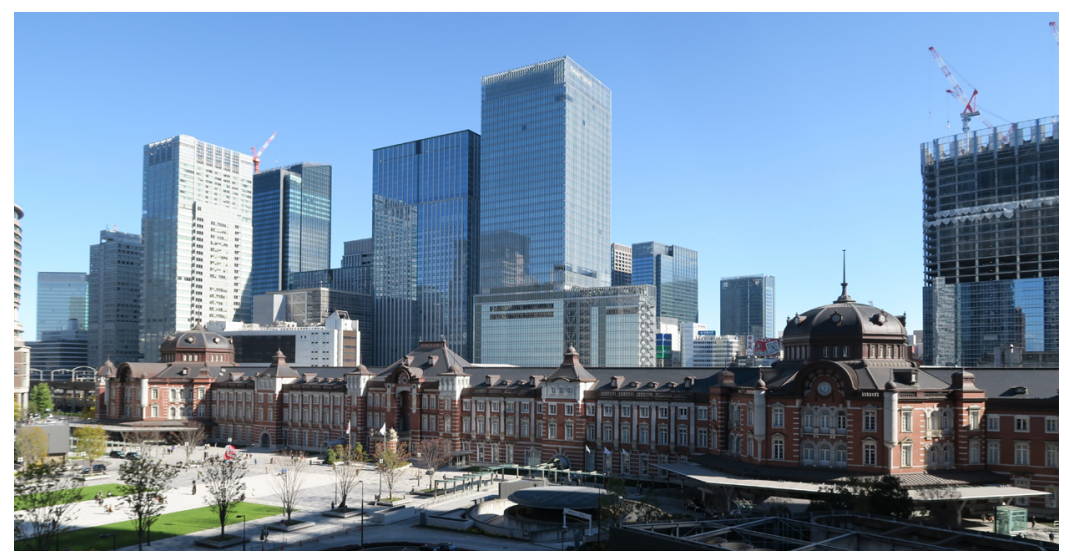

Fig. 03

Gare de Tokyo, 1914, par Tatsuno Kingo* 辰野金吾 ( 1854-1919).

Elle a été réhabilitée par le bureau d'architecture de la compagnie ferroviaire JR East (2012). La vaste esplanade blanche qui dégage la façade ouest se conforme au vocabulaire actuel du patrimoine historique en vigueur partout dans le monde.

Photo : Sylvie Brosseau 2020.

À une tout autre échelle, de nombreux jeunes architectes se consacrent à des projets de réhabilitation à partir de bâtiments modestes et ordinaires : maisons individuelles, apāto アパート (petits immeubles locatifs, souvent construits en bois sur deux niveaux, comprenant des logements d'une ou deux pièces). C'est par exemple le cas de Muraji Yutarō 連勇太朗 (né en 1987) qui a créé avec deux camarades Mokuchin kikaku モクチン企画, une organisation à but non lucratif basée à Tokyo et consacrée à la réhabilitation de ces bâtiments locatifs en bois, appelés mokuchin apāto, abréviation familière de mokuzō chintai apāto 木造賃貸アパート. Muraji en a fait la découverte en cherchant un local pour s'installer, une maison qu'il a réhabilitée où il vit et travaille. Il a alors commencé à observer ce patrimoine ordinaire avec un nouveau regard. Pour compenser la limite des interventions très ponctuelles sur la ville, est apparue l'idée de proposer en libre accès sur une plate-forme dédiée les projets réalisés et répertoriés, considérés en tant qu'outils de conception sous le nom de Mokuchin recipe, adaptables pour aménager son cadre de vie. Mokuchin kikaku a également publié plusieurs 
ouvrages présentant sa méthode ${ }^{23}$ et a engagé un partenariat avec une vingtaine d'agences immobilières. Muraji se place dans le sillage de Christopher Alexander ${ }^{24}$ (né en 1983, architecte et anthropologue américain d'origine autrichienne), qui, souligne-t-il, au lieu de mettre en valeur l'expression individuelle de l'architecte-auteur, a inventorié des figures modèles considérées comme des outils pour concevoir une architecture et des formes urbaines communes.

Muraji part du constat que la rénovation urbaine a transformé l'intérieur de la ligne Yamanote mais quautour, les bâtiments en bois restent nombreux (200 000 environ d'après ses recherches), et sont même prédominants dans certains quartiers. Ils reflètent un moment de l'histoire de la ville, quand la population affluait de province et que la croissance démographique amplifiait la pénurie de logements. Les propriétaires de maisons individuelles ou de petits terrains ont alors supplée au manque de logements et aux carences des pouvoirs publics en densifiant leur parcelle. Aujourd'hui, ces bâtiments sont considérés comme un "patrimoine négatif », perçus comme un risque potentiel en cas de séisme et d'incendie. Pour Mokuchin kikaku, ils constituent aussi un patrimoine culturel dont il faut prendre soin, diminuer la vulnérabilité, mais non pas le détruire. Muraji évoque un incendie particulièrement meurtrier à Shinjuku en 2011 qui a fait 18 victimes parmi les 23 résidents, des personnes âgées et pauvres. La réponse administrative consiste à résorber (selon la terminologie en vigueur) ce type d'habitat auquel colle une image de pauvreté. Mais, remarque Muraji, éliminer les bâtiments en bois ne résout pas la question sociale que cet incendie a révélée, celle de la grande pauvreté de nombreuses personnes âgées. Et ces logements bon marché constituent un filet de sécurité pour beaucoup de gens. D'où une offre de réhabilitation, projet complet ou bien très ponctuel, en fonction du budget disponible.

23. Le dernier opus est Kawase Hideya 川瀬英嗣 \& Muraji Yutarō, Mokuchin mesoddo : toshi o kaeru mokuchin apāto kaishū senryaku モクチンメソッドー都市を変える木賃アパート改 修戦略 (La méthode Mokuchin : stratégies de réhabilitation des appartements locatifs en bois pour changer les villes), Tokyo, Gakugei shuppansha 学芸出版社, 2017.

24. Alexander reprend et approfondit la théorie dite "pattern languages" (modèles ou motifs de conception comme solutions récurrentes), qui questionne l'idée de la création originale et individuelle. Les formes culturelles (artistiques, techniques ou autres) sont des types ou des modèles toujours actualisés dans les réalisations ou les œuvres individuelles qui sont donc issues d'un processus de création collectif. 
Mokuchin kikaku a pour objectif d'améliorer le confort et la sécurité de ces logements, ainsi que leurs espaces extérieurs par la création de connexions avec la rue et le voisinage; d'où une amélioration de l'image, donc de l'attractivité auprès d'une population nouvelle. Cette manière d'agir permet une " mise à jour" de la ville progressive sans effectuer de scrap and build radical, ni laisser la vacance et la dégradation s'enclencher. Muraji met en avant l'importance de ne pas effacer la mémoire du quartier, du bâtiment, mais souligne-t-il, cela n'a rien à voir avec le community design. Il s'agit de fabriquer une architecture qui permet d'établir des liens avec le quartier par des connexions physiques du bâtiment avec son voisinage, la rue, les habitants de l'immeuble, des connexions avec le temps en intégrant ce qui existe déjà, mais ce n'est pas à l'architecte de fabriquer les relations sociales ou la communauté.

Les quartiers denses, avec leurs ruelles, leurs jardinets ou leurs plantes en pots disposées devant les maisons, avec leur paysage caractérisé qui reflète un mode vie peu à peu idéalisé, sont depuis le début des années 2000 redécouverts de diverses manières. Par exemple Yanaka, où subsistent nombre de ces bâtiments ordinaires et longtemps déconsidérés, remarque Muraji, est devenu l'un des sites les plus fréquentés par les touristes, japonais ou étrangers.

Le même phénomène se déroule à l'est de la Sumida, au pied de la tour Sky Tree inaugurée en 2012. Ces quartiers populaires, moins onéreux, sont devenus les terrains d'expérimentation de nombreux jeunes architectes ou artistes qui installent leurs ateliers, toute une génération qui opère un retour à la fabrication et le revendique, comme une réaction au tout numérique, à la réalité virtuelle, à la dépossession par l'IA bientôt capable de faire un projet, au risque de répéter et renforcer les stéréotypes. Pour Muraji comme pour d'autres jeunes architectes de terrain, la pratique vient avant les concepts. C’est la monotsukuri sedai ものつくり世代, la génération qui fabrique.

Muraji, lors d'une table ronde ${ }^{25}$, ne s'est pas privé de tacler vigoureusement Ban Shigeru* 坂茂 (né en 1957), son professeur à l'université Keiō, qui expliquait à ses étudiants que les architectes ne travaillent que pour les classes supérieures possédant capital économique et culturel - et politique (l'architecture "haute couture " évoquée par Salvator-John A. Liotta et

25. Les informations sur Mokuchin kikaku proviennent de tables rondes avec Muraji Yutarō : en octobre 2017, organisées par les éditions LIXIL à Tokyo et en juillet 2019, par la villa Kujoyama à Kyoto. 
Aya Jazaierly). Les liens entre les architectes et la société sont coupés, d'où la nécessité des activités de bénévolat lors des catastrophes, seul moyen de les restaurer et de compenser certaines fautes. Muraji doute que le bénévolat dans un tel contexte favorise les relations entre les architectes et la société, il pense plutôt qu'il faut d'abord réfléchir sur le système et la structure qui produisent cette absence de lien, et essayer de les changer.

\section{Réémergence du bois}

L'architecture prémoderne japonaise est essentiellement construite avec des matériaux issus de végétaux, parmi lesquels le bois occupe une place structurelle capitale. Pendant des siècles, le Japon a vécu dans le bois, en a nourri sa culture. C'est encore le matériau de base utilisé par les charpentiers, les constructeurs de maisons individuelles, mais pendant longtemps, il reste éloigné de l'architecture moderne. Aussi étonnant que cela puisse paraître, la mise en valeur des usages du bois apparait comme une émergence importante de l'ère Heisei, cependant le choix actuel de ce matériau par les architectes reflète des attitudes diverses, et des situations non dépourvues d'ambiguïté.

Connu pour son travail avec le bois, Naitō explique qu'il a commencé à s'intéresser à ce matériau au début des années 1990 lorsque le conservateur du musée municipal de la Mer (Umi no hakubutsukan 海の博物館 ${ }^{26}$ ) à Toba dans le département de Mie, a souhaité utiliser ce matériau pour le bâtiment d'exposition, alors que les réserves venaient d'être achevées en béton ${ }^{27}$. La demande est donc venue du maître d'ouvrage et usager. Naitō s'est alors rendu compte que l'enseignement à l'université, focalisé sur le modernisme, sur l'acier et le béton, ne traitait pas du bois. Pour lui, ce choix a d'abord impliqué un apprentissage pour comprendre ses caractéristiques, le travail

26. Dans les années 1990, de nombreuses communes, même très petites, ont entrepris la construction de musées (autour d'une thématique régionale ou d'un artiste local) grâce à une subvention de cent millions de yens dans le cadre du " Furusato sōsei jigyō " ふるさと創生事業 (Plan de revitalisation du furusato) instauré en 1988 par le gouvernement du Premier ministre Takeshita Noboru 竹下登 (1924-2000). Ces nombreux projets publics ont atténué pour certains architectes les effets de l'éclatement de la bulle qui a tari la commande privée.

27. Grout, op.cit., p. 109. 
des charpentiers, puis réfléchir avec des ingénieurs à des systèmes de conception et fabrication par ordinateur afin d'optimiser les performances des structures, de les intégrer dans un cadre technologique contemporain. Les charpentes ainsi élaborées s'insèrent dans une tradition, se réapproprient une culture technique actualisée, rappellent que les matériaux de construction ont une origine et s'inscrivent dans une réalité historique. Pour Naitō, «ce choix répond à des exigences qui correspondent à sa manière de se penser et de se situer dans la société contemporaine japonaise à partir d'une critique de la modernité et d'une relation au contexte et aux usagers ${ }^{28}$ ". Rien n'est inné, n'a toujours existé, ni ne se perpétue naturellement, comme un patrimoine génétique, mais des savoirs et des techniques, fruits du travail, de pratiques, se transmettent, évoluent et se transforment ${ }^{29}$.

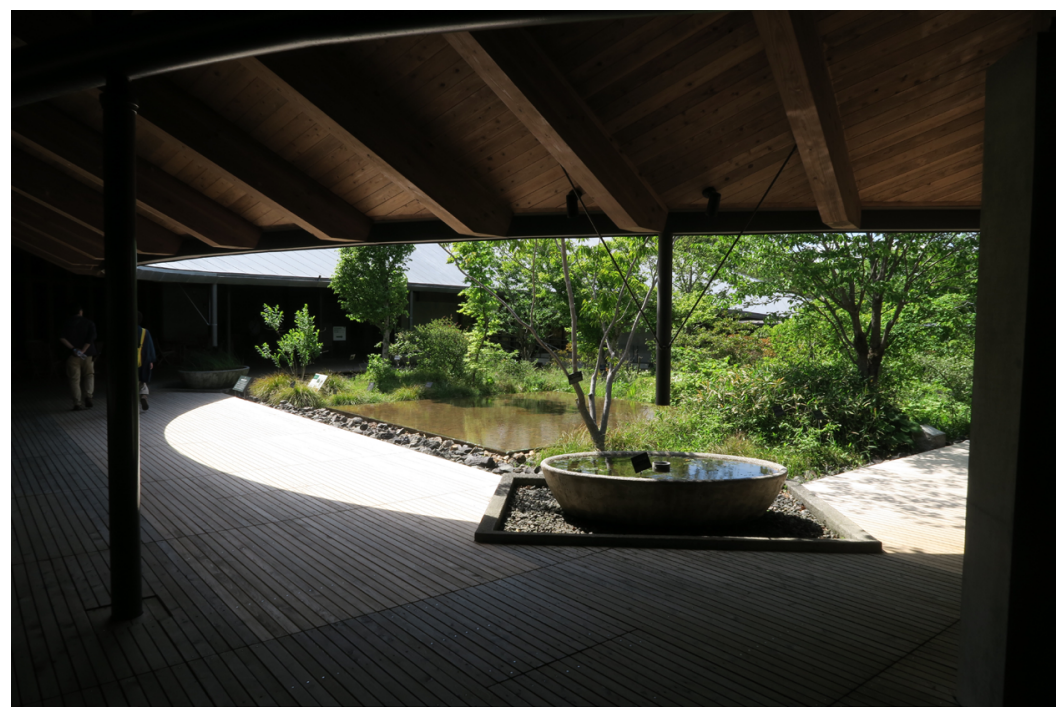

Fig. 04

Bâtiment du jardin botanique Makino de Kōchi, île de Shikoku (Kōchi kenritsu Makino shokubutsuen 高知県立牧野植物園), 1999, par Naitō Hiroshi.

Photo : Sylvie Brosseau 2019.

28. Id.

29. Jacquet Benoît, Matsuzaki Teruaki \& Tardits Manuel, Le charpentier et l'architecte, Lausanne, PPUR, 2019, p. 10. 
Un renouveau certain de l'architecture en bois au Japon est perceptible. Cependant, il faut souligner que celui utilisé dans la construction (que ce soit pour les matériaux composites, les lamellés-collés ou le bois brut) est peu ou pas produit au Japon, pourtant pays forestier majeur dans le monde. Le taux d'autosuffisance, de $100 \%$ dans les années 1950, est tombé à $18,8 \%$ en 2002 , pour remonter à $36,6 \%$ en 2018 . Le bois provient surtout de lieux de production bon marché, Asie du Sud-Est et Afrique, auquel s'ajoute le pin douglas nord-américain produit industriellement. Les matériaux composites comme le contreplaqué, utilisé pour les coffrages $\mathrm{du}$ béton, proviennent souvent d'abattages illégaux ${ }^{30}$. Le Japon participe donc à la déforestation de diverses régions du monde. Il faut rappeler aussi qu'une plantation d'arbres n'est pas une forêt (usage d'intrants chimiques, effet de la monoculture qui appauvrit les sols et réduit les écosystèmes viables de la faune sauvage). Tout reste à faire pour instaurer des cycles de production vertueux, alors qu'une des motivations importantes pour le choix du bois est d'intégrer la construction dans le cadre du développement durable, thème qui fait l'objet d'une prise de conscience à l'échelle planétaire. Comme souvent, on constate un écart entre l'image et l'impact réel des pratiques. De plus, la construction en bois au Japon souffre d'une législation anti-incendie très restrictive, d'où les difficultés persistantes de la filière.

Le choix d'utiliser du bois local a donc des raisons symboliques, éthiques ou économiques, par exemple pour soutenir la production régionale. C'est le cas du département de Kōchi dans l'île de Shikoku qui a sollicité Naitō à plusieurs reprises ${ }^{31}$ (fig. 04). La conception et la construction du bâtiment de l'EFEO à Kyoto, narrées par l'architecte Manuel Tardits, offrent un autre exemple de réflexion sur le choix des matériaux et leurs diverses significations ${ }^{32}$. Takamatsu, qui reconnaît que les préoccupations environnementales n'entraînent pas de changements notables dans son évolution conceptuelle, utilise le bois dans l'enveloppe de certains de ses édifices, et

30. "Ihō bassai no mokuzai, shinkokuritsu de shiyō fusegenu kanōsei NGO shiteki " 違法伐採の木材、新国立で使用防げぬ可能性 NGO指摘 (Bois illégalement abattu, une ONG observe que son utilisation pour le nouveau Stade national olympique ne peut être empêchée), Asahi shinbun 朝日新聞, 5 décembre 2016.

31. Grout, op.cit., p. 115.

32. Jacquet, Matsuzaki \& Tardits, op. cit., p. 295-367. 
va même jusqu'à proposer un projet de nouvel hôtel de ville à Kyoto sous la forme du plus haut bâtiment en bois du monde. Quant au stade olympique réalisé par Kuma avec l'entreprise Taisei, les plafonds des coursives nommés hisashi 庇 comme les auvents de l'architecture traditionnelle, sont habillés de lames en bois de sugi 杉 (Cryptomeria japonica) provenant des quarante-six départements du Japon ${ }^{33}$. Kuma explique qu'il est inspiré par la sagesse du satoyama 里山 ${ }^{34}$ et qu'il veut transmettre au monde, à travers le projet du stade olympique, ce modèle de coexistence entre la nature et les êtres humains ${ }^{35}$. Ces visions, esthétiques et idéalisées, convergent vers une nostalgie non dépourvue d'une affirmation identitaire.

\section{Pour clore Heisei, l’architecture japonaise s'expose}

Ces deux tendances, nostalgique et identitaire, ont trouvé une expression claire lors d'une grande exposition: "Japan in Architecture. Genealogies of its Transformation» ("Kenchiku no nihonten. Sono idenshi no motarasu mono » 建築の日本展。その遺伝子のもたらすもの) organisée à Tokyo par le Mori Art Museum du 25 avril au 17 septembre 2018, à l'occasion du quinzième anniversaire du complexe de Roppongi Hills et de son musée (fig. 05).

33. Le pin des Ryūkyū (Okinawa, $47^{\mathrm{e}}$ département) est réservé à un autre endroit.

34. Au cours de l'ère Heisei, le satoyama fait l'objet d'un intérêt qui s'est largement diffusé. Le terme, forgé par des écologues, désigne l'agrosystème propre au territoire rural prémoderne du Japon. Il est constitué d'une mosaïque de milieux, rizières humides et champs secs, jardins et vergers, étangs et ruisseaux, en lisière de bois ou de forêts. Produits de l'activité humaine, jusque dans les années 1950, ces bois étaient soigneusement entretenus en taillis, paysagés et même quasiment jardinés, essentiels pour leurs richesses économiques, culturelles et symboliques.

35. “Satoyama no chie gendai ni " 里山の知恵 現代に (La sagesse du satoyama, à notre époque), Mainichi shinbun 毎日新聞, 5 juin 2016. 


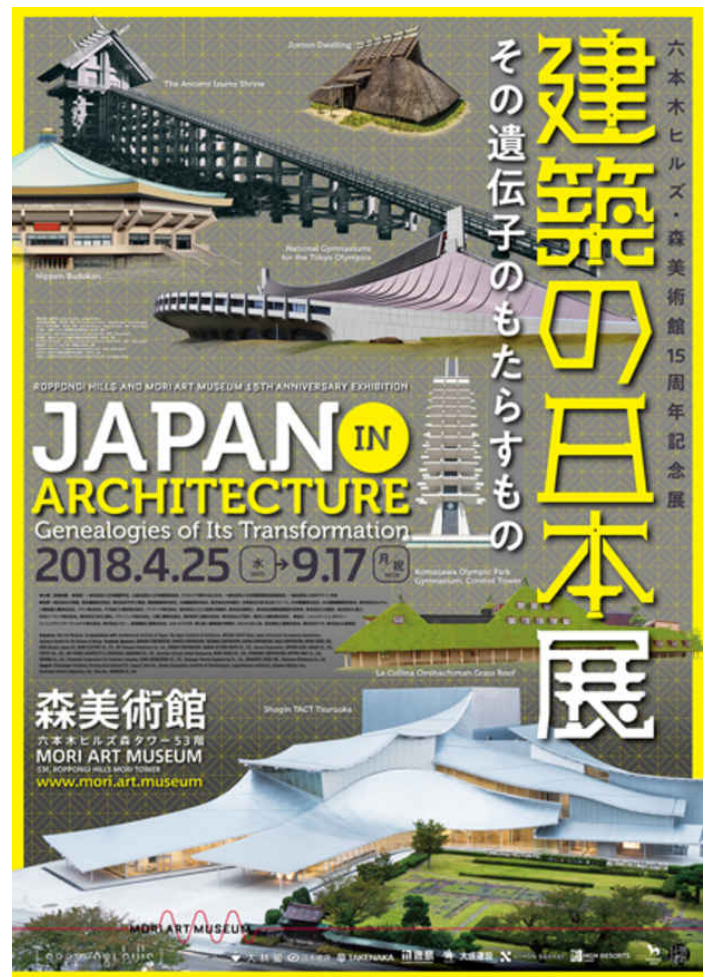

Fig. 05

Affiche de l'exposition « Japan in Architecture. Genealogies of its Transformation », Mori Art Museum, Tokyo, du 25 avril au 17 septembre 2018.

Cette exposition présentait une centaine de bâtiments ou ensembles architecturaux du Japon, embrassant toutes les époques, depuis l'ère mésolithique de Jōmon jusqu’à nos jours, avec plus de quatre cents documents, dont des vidéos, des installations, des maquettes parfois grandeur nature.

Le titre de l'exposition est donné en japonais et en anglais, comme tous les cartouches et panneaux, ainsi que le catalogue intégralement bilingue. Au-delà du public japonais, une audience internationale a d'emblée été visée, et le succès a été à la hauteur. En anglais, le mot genealogies est utilisé, alors qu'en japonais c'est idenshi 遺伝子 qui signifie gène. 
La même équivalence se retrouve dans l'avant-propos du catalogue rédigé par Nanjō Fumio 南條史生 (né en 1949), l'un des six curateurs et alors directeur du Mori Art Museum, qui présente ainsi l'exposition : l'architecture japonaise est aujourd'hui acclamée dans le monde entier et influente dans de nombreux pays. Cette exposition veut examiner ses caractéristiques et courants du point de vue de ses généalogies/gènes, de leur diffusion et expansion internationales actuelles. En explorant le passé et le présent de l'architecture japonaise, l'espoir est de contribuer à la construction d'" une nouvelle identité du pays " (Nihon toiu kuni no aratana aidentiti 日本という 国の新たなアイデンティティ $)^{36}$.

Le mot genealogies est systématiquement utilisé à la place de idenshi dans ce texte introductif, puis dans les autres articles du catalogue. Il ne s'agit donc pas, semble-t-il, d'une traduction approximative occasionnelle, mais bien d'un choix terme à terme. Les mots gène et idenshi renvoient à la génétique, à l'évolution biologique, à la nature, alors que la généalogie réfere à la société, à la transmission culturelle, à l'histoire.

Posé ainsi, l'objectif de cette exposition est, plutôt que l'étude des continuités et discontinuités historiques de l'architecture au Japon, la mise en évidence d'une évolution génétique. S'agit-il de prouver l'existence d'un ADN de l'architecture japonaise ainsi dévoilé? Ce terme d'ADN est d'ailleurs utilisé par divers auteurs, dont Fujimori Terunobu* 藤森照信 (né en 1946), dans certains textes du catalogue, avec pour résultat une naturalisation et une essentialisation du caractère nippon de l'architecture japonaise.

Pour préparer cette exposition majeure, le musée a réuni cinq curateurs, dont trois du Mori Art Museum, parmi lesquels le directeur (ce qui témoigne du niveau d'implication du musée), rejoints par Fujimori avec le rôle de conseiller, soit une équipe de six hommes japonais.

Le parti scénographique a choisi des effets visuels forts dans tout l'ensemble (photographies de grande taille, maquettes parfois imposantes, de bâtiments ou de détails, reconstitutions, installation laser...). Cependant, les documents présentés sans leur contexte historique ou culturel ont rendu cet ensemble foisonnant peu explicite. Labsence de profondeur historique

36. Mori Art Museum (dir.), Japan in Architecture. Genealogies of Its Transformation. Kenchiku no nihonten. Sono idenshi no motarasu mono 建築の日本展・その遺伝子のもたらす もの, Tokyo, Mori Art Museum/Echelle-1, 2018, p. 5. 
et le manque d'informations ont pour conséquence de donner à la plupart des pièces exposées le statut d'objets ou d'images, comme une collection de formes séparées de significations ou d'usages.

Le choix de présentation thématique, rejetant tout ordre chronologique, et donc la rupture moderne de l'ère Meiji, établit une continuité qui permet tout particulièrement de connecter l'ancien au présent, par un jeu de corrélations qui suggère des causalités.

La première et la dernière section de cette exposition, Possibilities of wood (Kanōsei toshite no mokuzō 可能性としての木造) et Living with nature (Kyōsei suru shizen 共生する自然), crée un cycle progressif qui part du bois, aboutit à la nature, et enveloppe l'exposition entière. La première salle, sous-titrée The soul that dwells in tree ( $K i$ ni semaru tamashii 木に泊る魂), présentait des maquettes du sanctuaire originel présumé d'Izumo (Izumo taisha 出雲大社), du sanctuaire d'Ise (Ise jingū 伊勢神宮), d'un pont-bâtiment monumental dessiné par Kuma (Yusuhara Wooden Bridge Museum [Yusuhara kibashi myūjiamu 梼原・木橋ミュージアム], 2010), et un fragment des assemblages en façade du pavillon du Japon à l'exposition universelle de Milan en 2015, conçu par Kitagawara Atsushi 北川原温 (né en 1951). Ce dispositif narratif révèle l'idée d'une architecture japonaise en harmonie avec la nature, fondée sur le bois. Le fameux "gène " qui porte l'ADN de l'architecture japonaise est le bois, conclut d'ailleurs Fujimori dans son article intitulé "Nihon, Sekai, Dentō, Modan»日本、世界、伝統、モダン (Japan, World, Tradition, Modern) ${ }^{37}$.

La dernière section de l'exposition remet l'accent sur l'harmonie avec la nature exprimée par l'architecture japonaise, grand mythe qui est présenté comme une caractéristique de l'" être japonais ». Un curateur du Mori Art Museum, Tokuyama Hirokazu 徳山拓一 (né en 1980), écrit qu'il faut regarder les œuvres architecturales du Japon comme des lieux pour dialoguer avec la nature, et y éprouver la vision de la nature enracinée dans le milieu japonais (Nihon no füdo 日本の風土). C'est, conclut-il, la fonction essentielle de l'architecture qu'aucun autre média ne peut remplacer ${ }^{38}$.

Qu'en est-il du musée et de son architecture? Quelle sorte de dialogue s'engage avec la nature et le milieu dans l'espace propre de cette exposition,

37. Ibid., p. 277.

38. Ibid., p. 265. 
c'est-à-dire le Mori Art Museum au 53 étage de la Mori Tower, siège social et vaisseau amiral de la Mori Building Company, située dans l'ensemble Roppongi Hills, un des plus vastes projets de rénovation urbaine du Japon, développé par la Mori Building Company de 2000 à 2003? Cette entreprise a été fondée en 1959 par Mori Taikichirō 森泰吉郎 (19041993), professeur d'économie à l'université de Yokohama, qui démarre en 1955 une affaire dans l'immobilier après avoir hérité de son père. Dans les années 1990, il devient l'homme le plus riche du monde. Mori s'est dédié au redéveloppement urbain de Tokyo, avec l'objectif de remplacer les constructions ordinaires en bois par des immeubles aux structures d'acier et de béton, de transformer les quartiers populaires de l'arrondissement de Minato en des centres urbains modernes constitués de smart buildings. Il était particulièrement fier du complexe Ark Hills, achevé en 1986, premier exemple de rénovation à grande échelle d'un quartier résidentiel ancien à flanc de colline, où est mise au point une " méthode Mori » de traitement de l'espace urbain.

Ce projet distribue autour d'un espace public ouvert du type dalle surélevée, des tours de bureaux, d'appartements de standing, un hôtel de luxe, auxquels sont adjoints un équipement culturel de rang mondial (la salle de concert Suntory Hall), le site d'un média (les studios de télévision de la chaîne Asahi) et des galeries marchandes. Mori Minoru 森稔 (1934-2012), diplômé de l'université de Tokyo, rejoint la société immobilière de son père et à la mort de celui-ci, devient à son tour président de Mori Building. Son projet le plus important est Roppongi Hills, sur le même modèle qu'Ark Hills, en plus grand et plus haut. Le dernier est Omotesando Hills (2006), un centre commercial controversé conçu par Andō le long de l'avenue Omotesandō, à la place d'un ensemble de logement social d'un grand intérêt historique et urbanistique. De petits immeubles, exemple d'une réflexion sur le logement collectif conçu et construit en 1926 par la Dōjunkai 同潤会, une fondation supervisée par le ministère de la Construction (Kensetsushō 建設省) après le tremblement de terre de 1923, ont donc disparu malgré les protestations. Depuis le décès de Mori Minoru, les activités de l'entreprise se poursuivent et le dernier grand projet en date est Toranomon Hills, achevé en 2014. Mori Minoru revendiquait l'influence de Le Corbusier* qu'il disait admirer et dont il collectionnait les dessins. Il pensait qu'il avait dépassé son maître dans la conception du projet de Roppongi Hills. Ce vaste et rutilant complexe a suscité de nombreuses critiques sur les 
destructions de Tokyo, de son paysage et de son échelle vernaculaires, sur la perte de la qualité de vie propre aux quartiers ordinaires où habitent des gens modestes, mais riches d'une subtile culture urbaine qui n'oublie pas la nature.

Au sommet de la Mori Tower, le Mori Art Museum a adressé, à travers cette exposition, divers messages portant sur les matériaux, l'esthétique, les valeurs, bref, a tenté de mettre en évidence par les œuvres exposées une japonité idéale, en contradiction avec le développement prôné et réalisé par la Mori Building Company. En d'autres termes, le contenu exposé dans le musée est à l'opposé de ce qui l'entoure.

Pour conclure, cette exposition, sous couvert d'expertise et de recherche d'une authenticité originelle, a revêtu une connotation idéologique certaine. La fluidité de l'espace et la continuité historique en viennent à être assimilées l'une à l'autre ${ }^{39}$. Les exemples architecturaux choisis, remodelés en objets symboliques par leur présentation et leur traitement, fabriquent une narration un peu trop cohérente. Celle-ci s'inscrit bien dans un mouvement de fond actuel et global, qui cherche à affirmer des racines culturelles, des gènes, des revendications identitaires, recrée un passé mythifié, et souffle un air aux effluves nationalistes.

L'ère Heisei a débuté dans le feu d'artifice d'une internationalisation qui semblait sans limite et finit dans le fantasme d'un furusato 古里 ${ }^{40}$ éventé. En ce sinistre début d'ère Reiwa 令和, n'est-ce pas la nostalgie de l'époque de la bulle que l'on sent frémir au Japon, comme le regret d'un monde irréel et festif, éclairé par des néons criards mais plus drôles que les opalescences pastel des leds?

Sylvie BROSSEAU Architecte-chercheuse, professeure à l'université Waseda à Tokyo et co-responsable du réseau scientifique thématique Japarchi (https://japarchi.fr).

39. Ibid., p. 277.

40. Furusato signifie "pays natal ", ville ou région d'origine qui reste chère au cœur. Ce terme cristallise de nombreux sentiments nostalgiques, évoque la désolation de la perte, ou l'idéalisation d'un lieu et d'un temps rêvés, ou encore les souvenirs de l'enfance, entre autres. Ces émotions sont aussi bien exprimées par la culture populaire (telle la chanson) que par la culture savante (telle la littérature), et ne sont pas exemptes de diverses instrumentalisations (par la publicité notamment). 